\title{
Design and Implementation of Micro-environment Intelligent Monitoring System in Computer Room based on Internet of Things Technology
}

\author{
Zhengqian Feng ${ }^{1,}$ a, Mingle Zhou ${ }^{1}$, Qingfeng Meng ${ }^{2}$ \\ ${ }^{1}$ Shandong Computer Science Center (National Supercomputer Center in Jinan), Shandong \\ Provincial Key Laboratory of Computer Networks, Jinan, China; \\ ${ }^{2}$ Shandong Computer Science Center, Shan Dong E-cloud Information Technology Co., Ltd., Jinan, \\ 250000, China \\ afengzhengqianf@126.com
}

Keywords: Computer room; Micro-environment; Monitor system.

\begin{abstract}
Data center microenvironment affects the reliable operation of servers and related network equipment, and it will also affect their performance and service life. But as more and more IT and electronic-equipment manufacturers are starting to improve technology, widely used "immersion silver" surface treatment technology in circuit board, which uses silver replace tin-lead alloy, the problems will follow. Because "immersion silver" treated board is easy to be corroded in high sulfur content and a certain humidity air environment. The silver and copper in the circuit board is etched, and derivatives produced gradually spread in the circuit board, which developing to a certain extent will cause electronic short circuit and then cause the device component failure. In order to ensure that the server device has a good operating environment, this article has designed a set of micro environment intelligent monitoring system, which can monitor and control the microenvironment and analyze the server operating environment and put forward relevant measures and suggestions for operational to ensure sustained and healthy operation of the IT systems in the computer room.
\end{abstract}

\section{Introduction}

Air quality in the computer room is a prerequisite for the normal operation of the communication device, Because if these devices run in the air quality exceeded for a long time is likely to result in decreased life of the equipment, the equipment plate corrosion, energy consumption increases, the failure rate rises and other issues, and may even lead to safety accidents.

Corrosive gases, especially hydrogen sulfide and sulfur dioxide are biggest impact on the safe operation of the communication device. Corrosive gases in the computer room will directly corrode equipment circuit board are running, especially the important silver plated circuit board if the concentration is too high. Over time, many of the key components and circuit boards of the master device will be corrosion failure or short circuit, resulting in device failure.

Because failures caused by air pollution are a large area, hidden and chronic cumulative, and such failures involve the entire room and difficult to find, the processing cycle is long, the lack of relevant guidelines maintenance personnel. In this case, people tend to ignore the security hidden danger. Therefore, when a situation occurs in the communication apparatus the usual practice is to replace the etched board only. But it is worth noting that if the air quality cannot be targeted to improve, even replacement of IT equipment, it still cannot avoid the disaster continue to occur. It's like a time bomb, and the ultimate victims are the user's business operations. Thus, the loss caused by the computer room air pollution is no less than any significant IT infrastructure failures.

Thus, in today with rapid development of the information industry, the Internet and communications industry, electronic information system room as an information hub has become the basis of all information systems. To ensure the stability and high availability of the computer room, electronic information system room not only reaches the standard and demand in the building or the acceptance, but also need monitor and manage of environmental parameters in the process of long-term operation and maintenance. 


\section{Current situation}

According to the degree of reaction of copper and gas pollution, air pollution levels can be obtained. For the classification of gas pollution degree, the current mainstream is shown in Table 1 and Table 2.

Table 1. the Classification of Environmental Pollution Degree

\begin{tabular}{|c|c|c|c|c|}
\hline Severity & G1 Weak & G2 Medium & G3 Strong & GX Serious \\
\hline Copper reaction degree( $(\grave{A})$ & $<300$ & $<1000$ & $<2000$ & $\geq 2000$ \\
\hline
\end{tabular}

Table 2. Gas Pollution Concentrations (Classification of Environmental Pollution Levels of the American Standard ISA-S71.04-1985) (ppm)

\begin{tabular}{|c|c|c|c|c|}
\hline GAS LEVEL & G1 Weak & G2 Medium & G3 Strong & GX Serious \\
\hline $\mathrm{SO}_{2} / \mathrm{SO}_{3}$ & $<0.01$ & $<0.1$ & $<0.3$ & $\geq 0.3$ \\
\hline $\mathrm{H}_{2} \mathrm{~S}$ & $<0.003$ & $<0.01$ & $<0.05$ & $\geq 0.05$ \\
\hline $\mathrm{Cl}_{2}$ & $<0.001$ & $<0.002$ & $<0.01$ & $\geq 0.01$ \\
\hline $\mathrm{NH}_{3}$ & $<0.5$ & $<10$ & $<250$ & $\geq 250$ \\
\hline $\mathrm{HF}$ & $<0.001$ & $<0.002$ & $<0.01$ & $\geq 0.01$ \\
\hline
\end{tabular}

Generally believed, level of G1 indicates that the data center environment is well controlled. It cannot consider the corrosion factor in determining the reliability of the equipment. When reaches the level of G2, corrosive effect of the environment can be considered to be measured, and it may be a factor in determining the reliability of the equipment.

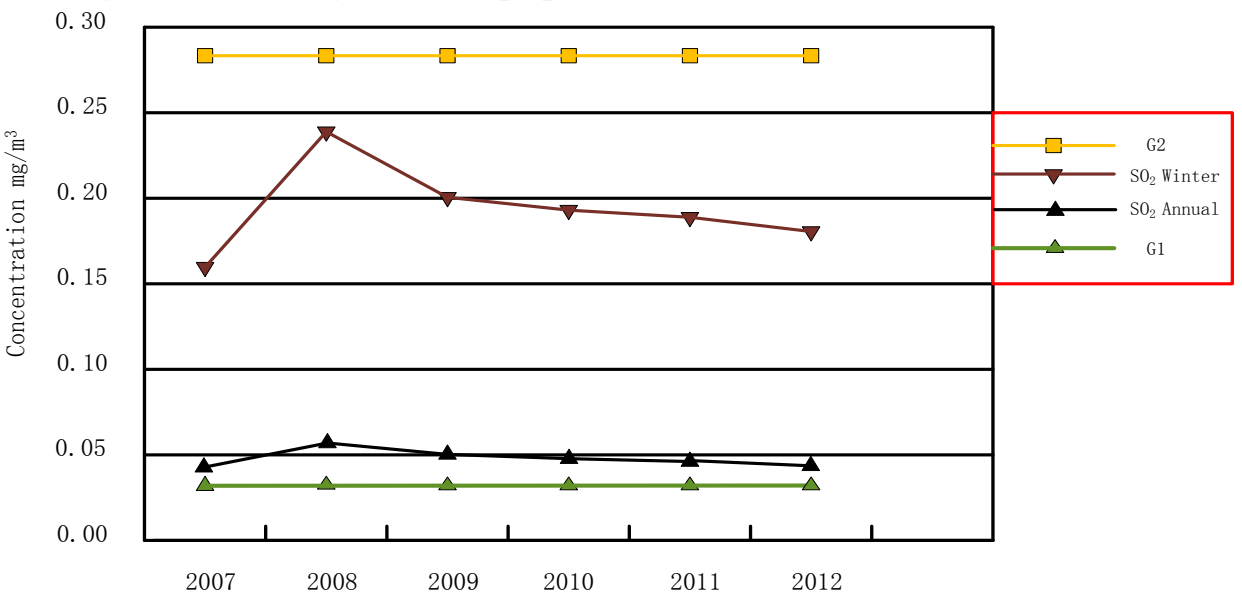

Fig1 the Annual Average Density of SO2 in Jinan Urban District

Figure 1 shows the average annual $\mathrm{SO}_{2}$ official data in Jinan City. Visible, data center in Jinan is in the air for a long time between G1 and G2 level.

Taking into account the above data measured is an average, it can be expected the average concentration is about 4 times in the winter. Therefore, as long as data center constructed in Jinan city, if you do not use air purification measures in the date room, $\mathrm{SO}_{2}$ may cause corrosion of IT equipment, especially in the winter. In this context, this paper designed a micro-environment intelligent monitoring system for the micro-environment monitoring and control in the date room.

\section{Solution}

When collecting the part of the data, it is not suitable using the way of the cable, such as collecting the data of temperature, humidity in the rack and the polluted gas in the computer room. That is because if used the manner of the cable, you should deploy the data line inside the rack, and it is bound to destroy the airflow causing the loss of refrigeration effect and increasing energy consumption. In extreme cases, it is likely because of the emergence of local hot spots causing equipment downtime. Therefore, in the computer room involved in this paper, the data collected try to give priority to using wireless-based to avoid the transformation of existing cabling room. Specifically, the solution is shown in Figure 2. 


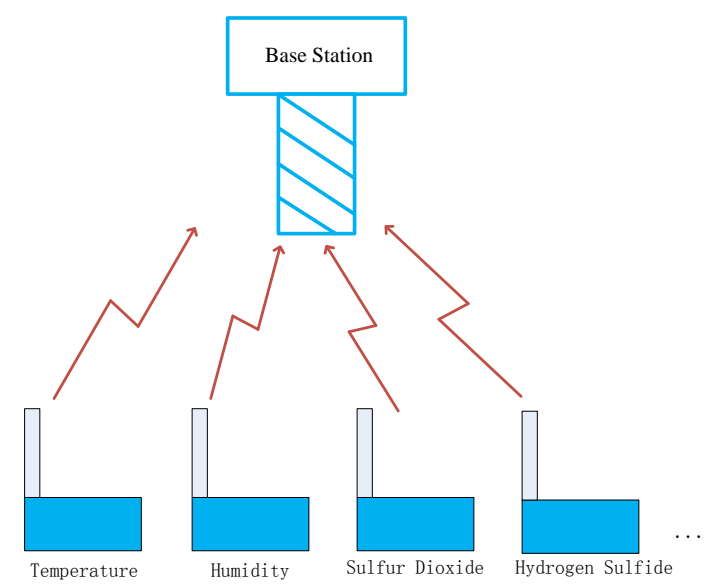

Fig 2 the Specific Solution

\section{System Deployment}

In order to implement the monitoring of air environment in the computer room, we deployed seven corrosive gas sensors. Among them, there are five sets of $\mathrm{SO}_{2}$ gas sensors and two sets of $\mathrm{NO}_{2}$ gas sensor. Deployment time is in June 2013, and deployment location is a computer room in Jinan. Specific deployment location is shown in Figure 3.

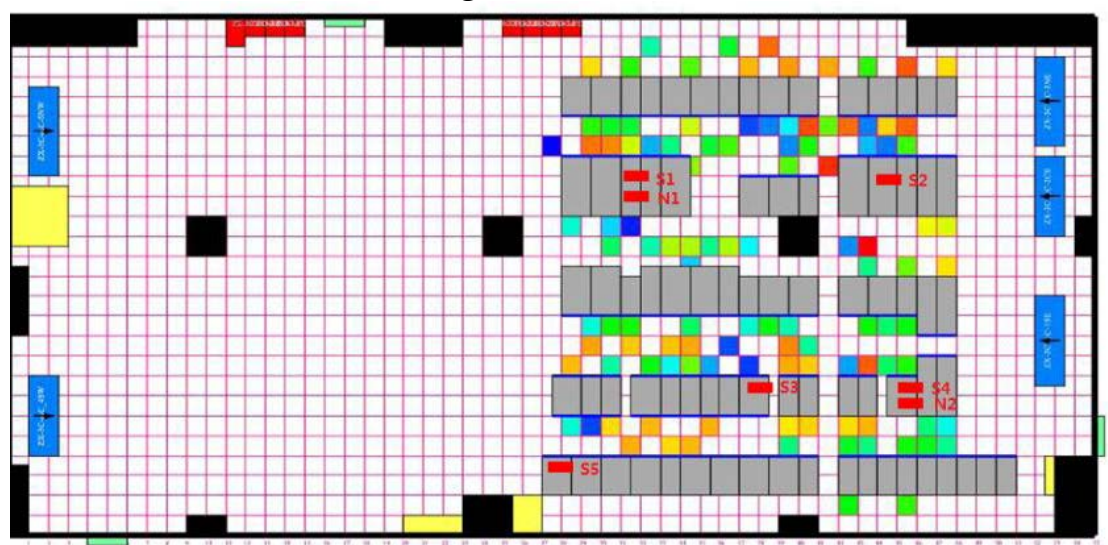

Fig 3 Sensor Deployment Diagram

In the figure, $\mathrm{S} 1, \mathrm{~S} 2, \ldots \mathrm{S} 5$ is $1 \#, 2 \# \ldots 5 \# \mathrm{SO}_{2}$ sensor respectively, $\mathrm{N} 1, \mathrm{~N} 2$ represents $1 \#$ and $2 \#$ NOx sensor.

\section{Test results}

Data recorded by the corrosive gases sensor in computer is shown in Figure 4 and Figure 5.

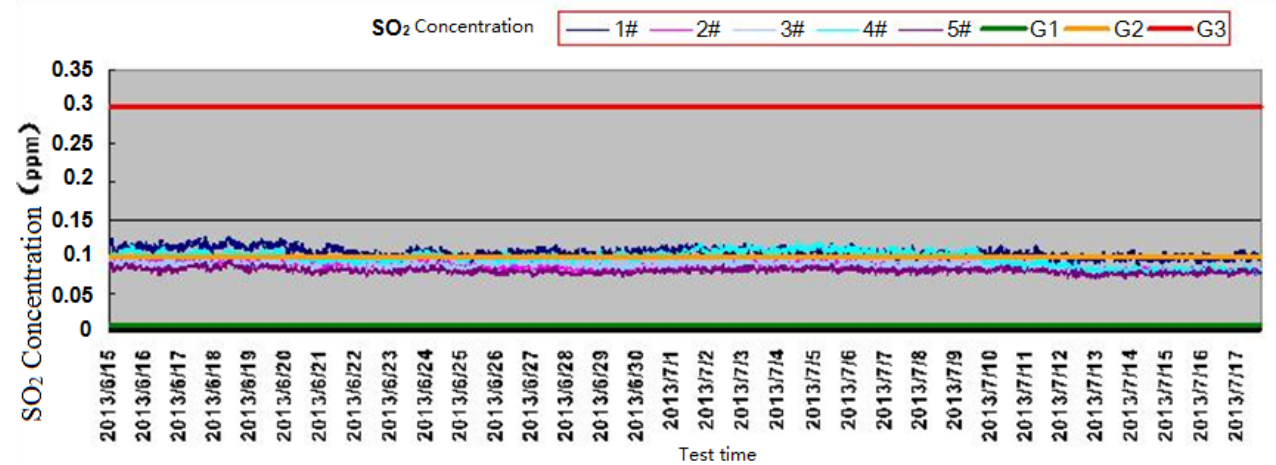

Fig 4 Monitoring Data of $\mathrm{SO}_{2}$

1 \#, 2 \#, ... 5 \# represent the data monitored by 1 \#, 2 \#, ... 5 \# SO 2 sensor. G1, G2 and G3 represent the level of G1 and G2 in ISA-S71.04-1985 standard. 


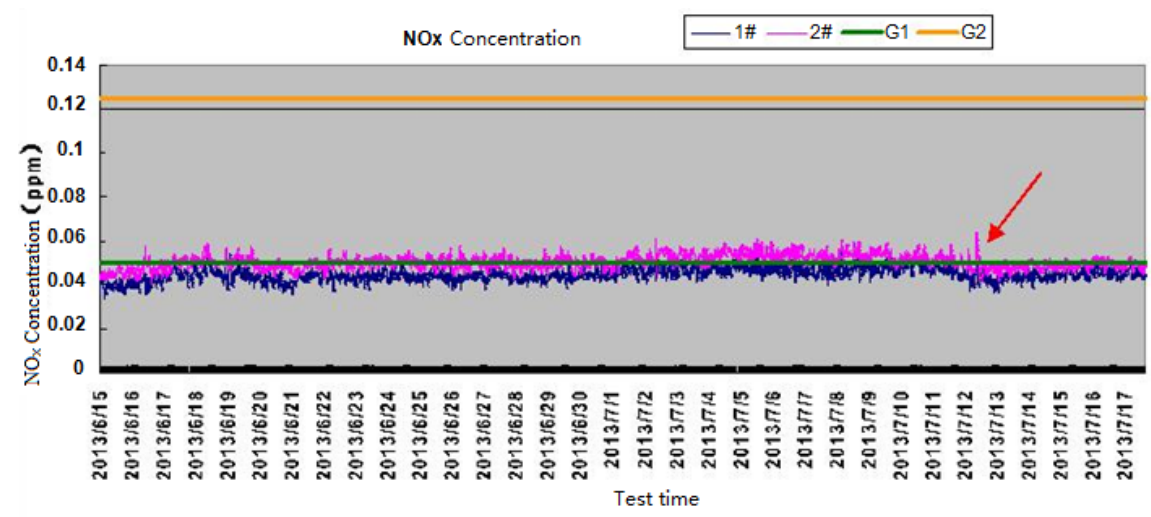

Fig 5 Monitoring data of $\mathrm{NO}_{2}$

1 \#, 2 \# represent the data monitored by 1 \#, 2 \# $\mathrm{NO}_{2}$ sensor. $\mathrm{G} 1$ and $\mathrm{G} 2$ represent the level of G1 and G2 in ISA-S71.04-1985 standard.

According to monitoring data in Figure 4 and Figure 5, the concentration of SO2 monitored is in the level between G2 and G3, and the concentration of NOx is in the level between G1 and G2. Thus it is concluded that, $\mathrm{SO}_{2}$ in the environment will cause corrosion damage to equipment, therefore, it is necessary for the staff to evaluate the environmental and further control the environment accordingly. For NOx in the environment, its corrosive effect is not too obvious.

\section{Conclusion}

The microenvironment monitoring system designed by this paper implements real-time monitoring $\mathrm{SO}_{2}$, temperature, humidity and other micro-environment in the air of the computer room. It helps data center operation and maintenance personnel to find sources of pollution, thus prompting them to take appropriate measures to meet the environmental requirements of equipment operation. Only in this way, the operation of the entire data center is safe and reliable. Therefore, it is important in the online monitoring of computer room microenvironment.

\section{References}

[1] Vigilant about "Hardware killer" in the computer room [J]. China computer world, 2009.08:1-2.

[2] Tang Zhiwen, Wang Qiang. Discussion on the Detection and Clean for Air Cleanness in Communication Room [J]. Telecom Power Technology. 2013, 30 (6) p.114-117.

[3] Liang Wei. Room power environmental monitoring and safety systems [J]. Friend of Science Amateurs. 2012.01p.154-155.

[4] Pan Kejia. Design and implementation of the computer room intelligent monitoring system based on internet of things technology [D]. ChengDu: University of Electronic Science and Technology of China, 2010.

[5] Sun Lan. Research of Environmental detection and evaluation method with Electronic information system room at operation and maintenance period [D]. TianJin: Tianjin University, 2012.

[6] Lin Ping. Environmental conditions of process measurement and control systems --Atmospheric pollutants [J]. China petrochemical Design. 1997, 14(2) p.61-65. 